\title{
Data-Driven Model Updating of an Offshore Wind Jacket Substructure
}

\author{
Dawid Augustyn ${ }^{\mathrm{a}, \mathrm{b}, *}$, Ursula Smolka ${ }^{\mathrm{c}}$, Ulf T. Tygesen ${ }^{\mathrm{b}}$, Martin D. Ulriksen ${ }^{\mathrm{d}}$, John D. \\ Sørensen ${ }^{2}$ \\ ${ }^{a}$ Department of the Built Environment, Aalborg University, Denmark \\ ${ }^{b}$ Ramboll Energy, Esbjerg, Denmark \\ ${ }^{c}$ Ramboll Energy, Hamburg, Germany \\ ${ }^{d}$ Department of Energy Technology, Aalborg University, Denmark
}

\begin{abstract}
The present paper provides a model updating application study concerning the jacket substructure of an offshore wind turbine. The updating is resolved in a sensitivity-based parameter estimation setting, where a cost function expressing the discrepancy between experimentally obtained modal parameters and model-predicted ones is minimized. The modal parameters of the physical system are estimated through stochastic subspace identification (SSI) applied to vibration data captured for idling and operational states of the turbine. From a theoretical outset, the identification approach relies on the system being linear and time-invariant (LTI) and the input white noise random processes; criteria which are violated in this application due to sources such as operational variability, the turbine controller, and non-linear damping. Consequently, particular attention is given to assess the feasibility of extracting modal parameters through SSI under the prevailing conditions and subsequently using these parameters for model updating. On this basis, it is deemed necessary to disregard the operational turbine states - which severely promote non-linear and time-variant structural behaviour and, as such, imprecise parameter estimation results-and conduct the model updating based on modal parameters extracted solely from the idling state. The uncertainties associated with the modal parameter estimates and the model parameters to be updated are outlined and included in the updating procedure using weighting matrices in the sensitivity-based formulation. By conducting the model updating based on in-situ data harvested from the jacket substructure during idling conditions, the maximum eigenfrequency deviation between the experimental estimates and the model-predicted ones is reduced from $30 \%$ to $1 \%$.
\end{abstract}

Keywords: model updating, operational modal analysis, jacket substructure, wind turbines, digital twin, condition monitoring

\section{Introduction}

The offshore wind industry has experienced strong growth over the last decade [1]. As a result, the cumulative capacity of installed offshore wind turbines has reached $23 \mathrm{GW}$ in 2018 with additional $40 \mathrm{GW}$ planned to be operational within the next 5 years [2]. Building upon a commercial success of this technology, more structures are being installed and instrumented with measurement equipment, thus allowing to capture more operational information. In fact, some authorities, including the German certification body Federal Maritime and Hydrographic Agency, have already requested new structures to be equipped with monitoring systems [3].

Access to information from monitoring systems can bring vital insight into a revised, more accurate estimate of the condition of the structures. Given proper processing, such insight can be used to optimize the operation of existing structures and to improve design procedures for structures to follow. A specific approach is to use the concept

\footnotetext{
${ }^{*}$ Corresponding author. Ramboll Energy, Esbjerg, Denmark

Paper published in Applied Ocean Research, doi: https://doi.org/10.1016/j.apor.2020.102366

Email address: dawa@ramboll.com (Dawid Augustyn)
} 
of a digital twin [4], where operational data is used to calibrate numerical models to reflect, in the best possible way according to some performance measure, the behaviour of a physical asset. The digital twin concept is well established and widely used in many industries, i.a., aerospace engineering [5] and the oil and gas industry [6]. Recently, a digital twin has also been presented as a key element for establishing a data-driven conceptual framework for enhanced operation of offshore wind structures [7]. Therein the model updating methods are implemented to establish a digital twin, which is subsequently used to perform lifetime extension, either 1) deterministically or 2) probabilistically, as illustrated in Fig. 1.

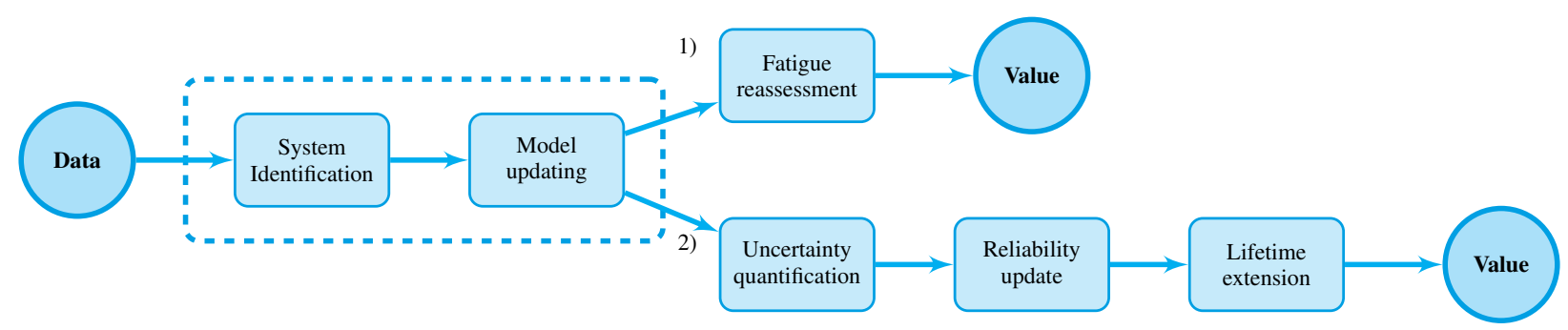

Figure 1: Application of a digital twin for lifetime extension [7]. The dashed rectangle highlights the scope of this paper.

To establish a digital twin, one normally starts with developing an initial model based on a generic estimation of the physical parameters. Afterwards, operational data is collected and features extracted and compared to model predictions. Typically, one compares a subset of modal parameters as it can be conveniently estimated from operational data by means of system identification methods. Some full scale validations of system identification methods have been performed for onshore wind structures [8], offshore platforms [9], offshore wind tripod substructures [10], and offshore wind monopile substructures [11]. However, limited publications exist that explore the applicability of the system identification methods for offshore wind jacket substructures. The challenge for this type of substructures lies in the dynamic blade-substructure coupling [12] and in the non-linear behaviour of the controller.

Once the discrepancy between a physical structure and its digital counterpart is quantified, model updating methods can be used to reduce the difference by updating selected physical parameters of the numerical model [13, 14]. Again, various methods have been validated for aerospace and automotive applications $[15,16]$ and for wind turbine blade structures [17]. However, to the authors' knowledge, no dedicated application study has been presented for updating offshore wind turbine substructures.

The present paper aims to address the above-mentioned research gap by applying a model updating framework in the context of an offshore wind jacket substructure. The contributions of this paper are; 1) applying the model updating framework based on in-situ data (including system identification results) and 2) providing recommendations on practical implementation of the framework.

\section{Methodology}

In this section, the theoretical background for the methods used in the model updating framework is presented. Well-established procedures for both system identification and model updating are summarized, followed by a discussion on the validity of the main assumptions in the context of offshore wind applications.

\subsection{System identification}

The covariance-based stochastic subspace identification (SSI-cov) method is used to estimate modal parameters of the wind turbine jacket substructure [18]. The merits of the SSI-cov method include its simplicity in implementation and numerical robustness [18]. The former merit is especially attractive from a user perspective as the method requires only a model order as input, while the remaining parameters can be estimated accordingly. The numerical robustness implies that no issues with convergence arise, hence making the method applicable for large structures and data sets. Consequently, this particular method is extensively applied in the offshore wind and oil and gas industries [19, 8, 6, 10]. 
Consider the state-space formulation of a linear, time-invariant (LTI) system

$$
\left[\begin{array}{c}
\mathbf{x}_{i+1} \\
\mathbf{y}_{i}
\end{array}\right]=\left[\begin{array}{c}
\mathbf{A} \\
\mathbf{C}
\end{array}\right] \mathbf{x}_{i}+\left[\begin{array}{c}
\mathbf{w}_{i} \\
\mathbf{v}_{i}
\end{array}\right],
$$

where $\mathbf{x}_{i} \in \mathbb{R}^{n}$ and $\mathbf{y}_{i} \in \mathbb{R}^{m}$ are the state and output vectors for time instance $t_{i} . \mathbf{A} \in \mathbb{R}^{n \times n}$ and $\mathbf{C} \in \mathbb{R}^{m \times n}$ are the state and output matrices, respectively. The unknown input $\mathbf{w}_{i} \in \mathbb{R}^{n}$ and measurement noise $\mathbf{v}_{i} \in \mathbb{R}^{m}$ are modelled as discrete, white-Gaussian sequences with zero mean; thus, $\mathbf{w}_{i} \sim \mathcal{N}\left(\mathbf{0}, \boldsymbol{\Sigma}_{W}\right)$ and $\mathbf{v}_{i} \sim \mathcal{N}\left(\mathbf{0}, \boldsymbol{\Sigma}_{v}\right)$ with the following covariance matrices:

$$
\mathbb{E}\left[\left(\begin{array}{c}
\mathbf{w}_{p} \\
\mathbf{v}_{p}
\end{array}\right) \quad\left(\begin{array}{ll}
\mathbf{w}_{q}^{T} & \mathbf{v}_{q}^{T}
\end{array}\right)\right]=\left[\begin{array}{cc}
\boldsymbol{\Sigma}_{\mathbf{w}} & \boldsymbol{\Sigma}_{\mathbf{w v}} \\
\boldsymbol{\Sigma}_{\mathbf{w v}}{ }^{T} & \boldsymbol{\Sigma}_{\mathbf{v}}
\end{array}\right] \delta_{p q} \succcurlyeq 0,
$$

where $\delta_{p q}$ is the Kronecker delta, $\boldsymbol{\Sigma}_{w} \in \mathbb{R}^{n \times n}, \boldsymbol{\Sigma}_{v} \in \mathbb{R}^{m \times m}$, and $\boldsymbol{\Sigma}_{w v} \in \mathbb{R}^{n \times m}$. The input term and measurement noise are assumed to be spatially and temporally uncorrelated.

Let $\mathbf{Y}_{i \mid i} \in \mathbb{R}^{m \times j}$ be the output Hankel matrix and $\hat{\mathbf{X}}_{i} \in \mathbb{R}^{n \times j}$ a state sequence estimate, then the state-space matrices can be estimated as

$$
\left[\begin{array}{c}
\hat{\mathbf{A}} \\
\hat{\mathbf{C}}
\end{array}\right]=\left[\begin{array}{c}
\hat{\mathbf{X}}_{i+1} \\
\mathbf{Y}_{i \mid i}
\end{array}\right] \hat{\mathbf{X}}_{i}^{\dagger},
$$

where superscript $\dagger$ denotes Moore-Penrose pseudo-inverse and $n_{s}$ is the selected model order. From $\hat{\mathbf{A}} \in \mathbb{R}^{n_{s} \times n_{s}}$ and $\hat{\mathbf{C}} \in \mathbb{R}^{m \times n_{s}}$, the eigenfrequencies, $f_{i}$, damping ratios, $\zeta_{i}$, and mode shapes, $\phi_{i}$, can be estimated.

The SSI-cov method hinges on a number of assumptions, including 1) the system is LTI and 2) the input is ergodic, white, Gaussian, and spatially and temporally uncorrelated. The validity of the assumptions for offshore wind applications is briefly discussed below.

In practice, the LTI assumption is always violated, as each system changes its properties with respect to both loading conditions and time. For offshore wind applications, the non-linear behaviour is mostly introduced due to the turbine controller, and to a lesser degree by the soil behaviour. A turbine controller is constantly adjusting turbine properties (pitch, yaw) to harvest the maximum amount of energy. Such adjustments alter the dynamic properties of the system, which leads to varying modal parameters.

The SSI-cov method requires no prior knowledge on the input. Effectively it is assumed ergodic, white-noise Gaussian, and spatially and temporally uncorrelated. Assuming the input emulates the turbine's exposition to environmental conditions it is evident that wind and wave properties can change from one sea state to another, thus violating the ergodicity property. Moreover, the frequency spectrum originating from wind and wave excitation is far from being white-noise Gaussian due to rotating machinery, the peak period in the wave spectrum, and background turbulence in the wind spectrum.

Finally, some major assumptions are made with respect to damping. In Eq. 1, the damping is assumed to follow a linear viscous model, which is typically established on the premise of the distribution being classical. This is, by no means, a fully representative model of the damping sources present in offshore wind applications, e.g., radiation soil damping, hydrodynamic damping, and aerodynamic damping.

The above-mentioned assumptions are, in principle, violated for all practical applications. Nevertheless, useful results for estimating modal parameters are reported for, e.g., offshore wind monopile turbines [19], onshore turbines [8], and offshore oil and gas jacket platforms [6]. On that basis the applicability of the SSI-cov system identification method in the context of an offshore wind jacket substructure is explored in this paper.

\subsection{Sensitivity-based model updating}

Model updating techniques can be used to update a set of selected physical parameters of the model to better reflect the in-situ conditions. The sensitivity-based method, widely used in adjacent industries [15], is implemented here. Below, key elements of the theoretical framework are presented, followed by a discussion of the assumptions made. For a comprehensive derivation of the model updating method, the reader is referred to [20].

We define the discrepancies in modal responses, $\Delta \boldsymbol{\Lambda}$, as a difference between estimated responses, $\boldsymbol{\Lambda}_{S}$, and numerical predictions, $\boldsymbol{\Lambda}_{\mathcal{M}(\Theta)}$. In this study, eigenmodes and eigenfrequencies are used as modal responses. $\Delta \boldsymbol{\Lambda}$ can be approximated by the linear Taylor expansion

$$
\Delta \mathbf{\Lambda} \approx \mathbf{S} \Delta \boldsymbol{\Theta},
$$


where $\Delta \boldsymbol{\Theta}$ contains the parameter shifts to be estimated and $\mathbf{S}$ is the sensitivity/Jacobian matrix, thus

$$
S_{j k}=\left[\frac{\partial \Lambda_{j}}{\partial \Theta_{k}}\right],
$$

where $\Lambda_{j}$ is the $j$ 'th response and $\Theta_{k}$ is the $k^{\prime}$ th parameter. The sensitivity matrix is obtained via perturbations directly from the numerical model.

The measurements and response estimates are inherently corrupted with uncertainties. These uncertainties are included in the updating procedure, as elaborated in Subsec. 2.3. In many applications, the number of parameters to be updated is larger than the number of responses identified from the physical structure. This poses a central problem for model updating in engineering applications, as the solution obtained is non-unique. To address this problem, a regularization scheme, originating from Tikonov [21], is applied in the paper to add a penalty on the objective function. A regularization coefficient, $\alpha$, is included in the formulation of the objective function to minimize the weighted parameter change.

The objective function accounting for the above-mentioned extensions is defined as

$$
J=\Delta \mathbf{\Lambda}^{T} \mathbf{W}_{\Lambda} \Delta \mathbf{\Lambda}+\alpha \Delta \boldsymbol{\Theta}^{T} \mathbf{W}_{\Theta} \Delta \boldsymbol{\Theta}
$$

where $\mathbf{W}_{\Lambda}=\operatorname{diag}\left(\sigma_{\Lambda_{j}}^{2}\right)^{-1}$ is a diagonal weighting matrix expressing uncertainties in the responses and $\mathbf{W}_{\Theta}=$ $\operatorname{diag}\left(\sigma_{\Theta_{k}}^{2}\right)^{-1}$ is a diagonal weighting matrix expressing uncertainties in the model parameters. The weighting coefficients $\sigma_{\Lambda}^{2}, \sigma_{\Theta}^{2}$ include variance of responses and parameters, respectively. The optimal parameter set is obtained iteratively by minimizing the objective function in Eq. 6 , $\arg \min _{\Delta \Theta} J$, hence

$$
\boldsymbol{\Theta}_{i+1}=\boldsymbol{\Theta}_{i}+\left[\mathbf{S}_{i}^{T} \mathbf{W}_{\Lambda} \mathbf{S}_{i}+\alpha \mathbf{W}_{\Theta}\right]^{\dagger} \mathbf{S}_{i}^{T} \mathbf{W}_{\Lambda} \Delta \mathbf{\Lambda}_{i}
$$

The regularization ensures that the parameter changes are minimized. It is assumed that parameter uncertainties are uncorrelated. Nevertheless, fully populated covariance matrices could easily be included, if correlation between parameters is deemed critical, e.g., correlated mass or stiffness parameters.

\subsection{Uncertainties included in the updating procedure}

The main types of uncertainties related to the parameters, $\boldsymbol{\Theta}$, and modal response estimations, $\boldsymbol{\Lambda}_{s}$, are summarized below along with their implementation in the updating procedure. Quantification of the uncertainties, based on engineering judgement, is provided in Sec. 5.

Uncertainties in the context of modal updating can be divided into two categories: 1) aleatory, related to the natural variation in a physical system (stiffness, mass, and damping), and 2) epistemic, related to lack of knowledge describing a system (modelling quality, statistical uncertainties, and measurement quality). Once the system is realized and some measurands are captured, aleatory uncertainty can be reduced with the expense of the additionally introduced (epistemic) uncertainties, e.g., stiffness/geometry becomes deterministic within the precision of the measurement equipment. The premise of model updating for fatigue reassessment is that the gain in reducing aleatory uncertainty is larger than the additional epistemic uncertainties, thus improving the final model. Below, some examples of uncertainties introduced in the updating procedure are listed, following the uncertainty definition provided in [22]. Aleatory uncertainty (for the context of this paper) stems from inherent variation in physical parameters describing stiffness, damping, and, to a lesser degree, mass of a system. These quantities are modelled by prior (generic) distribution functions of the physical parameters assumed at the design stage. During the updating procedure, these distribution functions are substituted with most likely mean values based on novel (in-situ) information from the operational stage. The aleatory uncertainties are included in the updating procedure in the weighting matrix $\mathbf{W}_{\Theta}$ as described in Subsec. 2.2 and Subsec. 5.3.

Epistemic uncertainties stem from data processing and are, in the framework of model updating, realized as measurement, statistical, and model uncertainties. Measurement uncertainties are governed by the quality of the sensor setup (sensor type and sensor placement). These uncertainties affect the number of dynamic modes that can be quantified. Typically, a sensor setup is a balance between practical limitations and the targeted quality of the information 


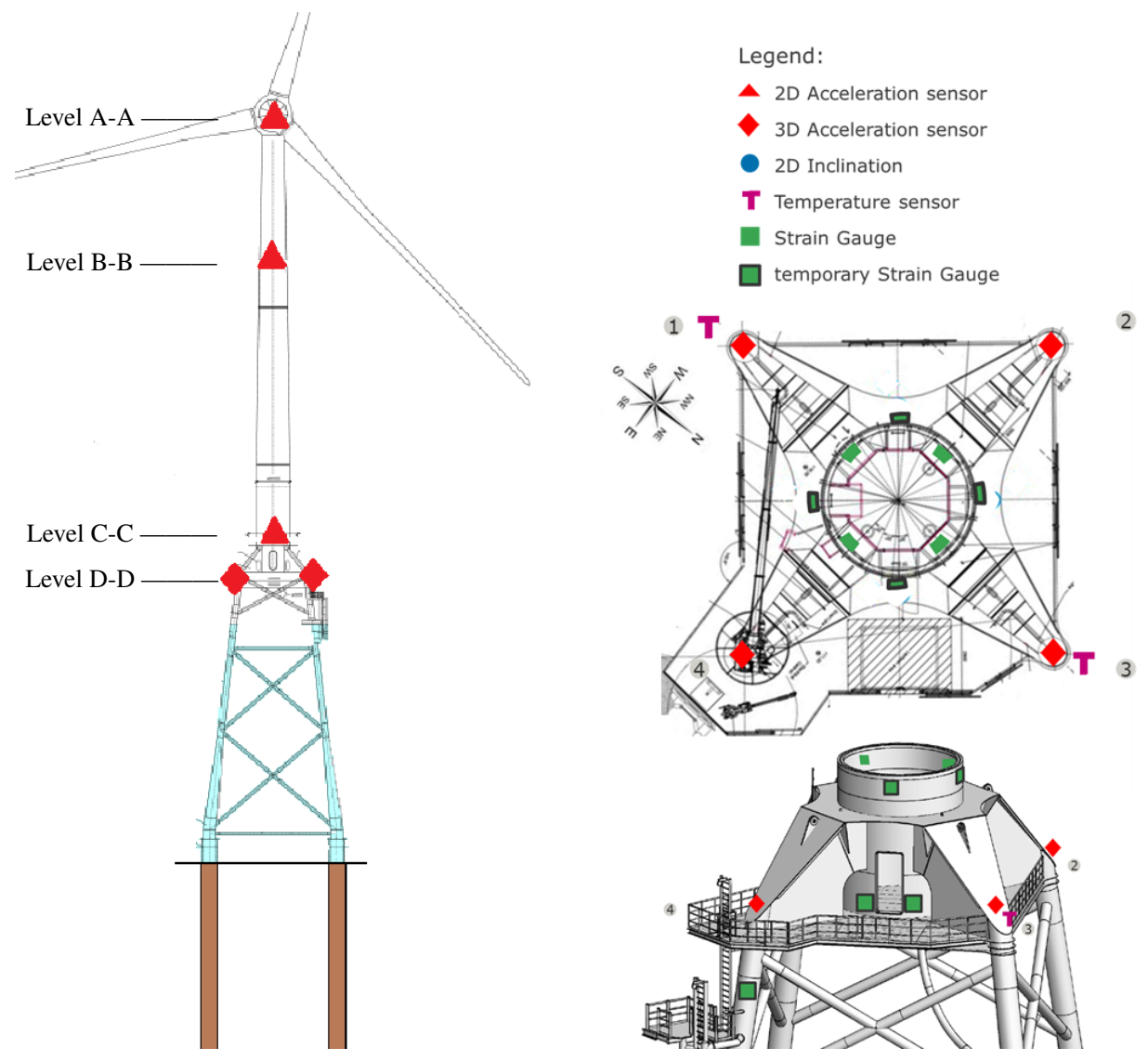

Figure 2: Wind turbine geometry and measurement setup.

extracted. Moreover, this uncertainty accounts for signal noise and various pre-processing steps. Statistical uncertainties are related to the amount of data captured. A data set should be sufficiently large to capture a statistically sound estimation of the variation (uncertainties) in the modal parameters extracted. Model uncertainties are related to the method and algorithm used in the updating procedure, including the system identification. For SSI, the associated uncertainties can be quantified as described in, e.g., [23]. The summarized epistemic uncertainties are included in the updating procedure in the weighting matrix $\mathbf{W}_{\Lambda}$ as described in Subsec. 2.2 and Subsec. 5.2.

The updating procedure described in Subsec. 2.2 aims at improving the accuracy of a numerical model by updating the mean values of selected physical parameters in the presence of uncertainties. Thus, the uncertainties are not updated but merely serve as input to $\mathbf{W}_{\Lambda}$ and $\mathbf{W}_{\Theta}$. Updating of the uncertainties can be performed by, e.g., Bayesian methods; however, this is outside of the scope of this paper.

\section{Measurement campaign}

The model updating framework is applied in the context of a measurement campaign within the $350 \mathrm{MW}$ Wikinger wind farm. The farm consists of 70 Adwen $5 \mathrm{MW}$ wind turbines located in the German exclusive economic zone of the Baltic Sea. Measurements from one turbine are used to perform model updating. The chosen wind turbine is placed on a 4-legged jacket substructure, see Fig 2 . The hub height (A-A) of the wind turbine is approximately $90 \mathrm{~m}$ above the mean sea level (MSL). The interface level between the transition piece (TP) and the wind turbine tower is approximately $18 \mathrm{~m}$ above MSL (C-C), and the tower length is approximately $72 \mathrm{~m}$. The water depth at the location of the instrumented wind turbine is approximately $38 \mathrm{~m} \mathrm{MSL}$, the jacket height (including TP) is approximately 54 $\mathrm{m}$, and the pile penetration depth is approximately $30 \mathrm{~m}$ beneath the sea bed. The soil is mainly considered as clay. 
a) 1 st bending mode

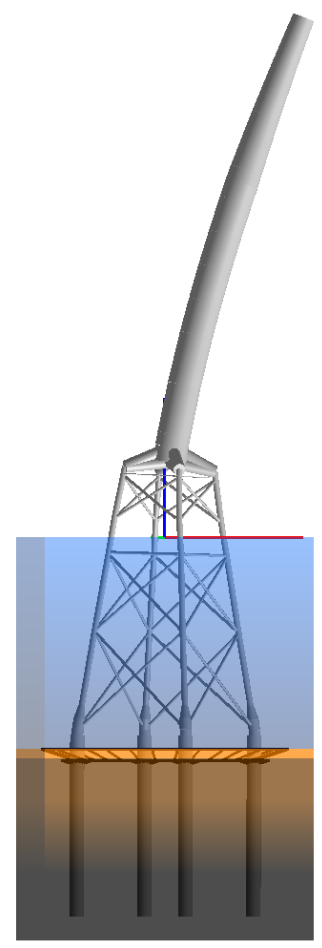

b) 2nd bending mode

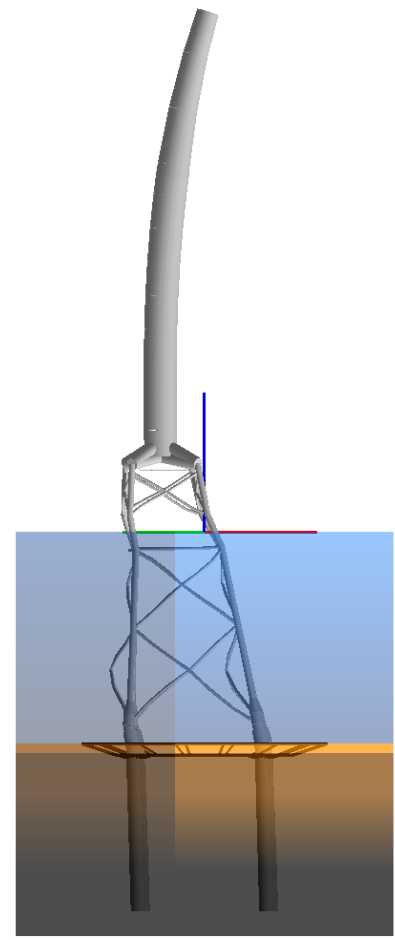

Figure 3: Global eigenmodes (fore-aft/side-side): a) 1st bending, b) 2nd bending.

The measurement data used for model updating is composed of acceleration signals captured over a period of 1 month. The sensor setup includes 4 triaxial accelerometers at the TP (section D-D, one accelerometer per leg), 1 biaxial accelerometer installed at the tower bottom (section $\mathrm{C}-\mathrm{C}$ ), 1 biaxial accelerometer in the tower, approximately at $2 / 3$ of the height (section B-B), and 1 biaxial accelerometer at the tower top (section A-A). The approximate locations of the sensors are depicted in Fig. 2. The chosen configuration is primarily aimed at identification of the first 5 global dynamic modes, including 2 global bending modes (both in fore-aft and side-side direction) and a torsional mode. A sketch of the 1st and 2nd global bending modes is presented in Fig. 3. Fig. 3a shows the 1st global bending mode in both fore-aft and side-side directions, where major deformation is observed in the tower of the structure. These modes correspond to eigenmodes 1 and 2 of the structure. Fig. $3 b$ shows the 2 nd global bending mode in foreaft and side-side directions, where major lateral deformation is observed in the TP level. These modes correspond to eigenmodes 4 and 5 of the structure. Note that eigenmode 3, which is the first torsional mode, is not presented here.

In addition to the acceleration signals, a subset of SCADA (supervisory control and data acquisition) data is also recorded. The turbine data and ambient wind data are collected as 10-min statistics. In Fig. 4, some SCADA data are presented to visualize the wind speed and operational state of the turbine during the measurement campaign. The recorded wind speeds span from $0 \mathrm{~m} / \mathrm{s}$ to slightly above $20 \mathrm{~m} / \mathrm{s}$. Within this range, the turbine states are divided into 3 categories: 1) idling or parked state where aerodynamic and rotor forces are reduced, 2) operational state below the rated power with the blades pitching and the rotor altering its angular velocity, and 3) operational state with the blades pitching to keep the angular velocity of the rotor and the power output constant. The cut-in and rated wind speeds, i.e., $U_{c}=4 \mathrm{~m} / \mathrm{s}$ and $U_{r}=12 \mathrm{~m} / \mathrm{s}$, respectively, are depicted in Fig. 4 with dashed lines. The turbine states are categorized based on the power output, which is more precise compared to relying solely on the wind speed. This can be noticed in Fig. 4, as in some cases the rated power is reached for wind speeds below $12 \mathrm{~m} / \mathrm{s}$. Moreover, in other cases, the wind turbine is idling despite the wind speed exceeding the cut-in value, which can be due to, e.g., maintenance. 


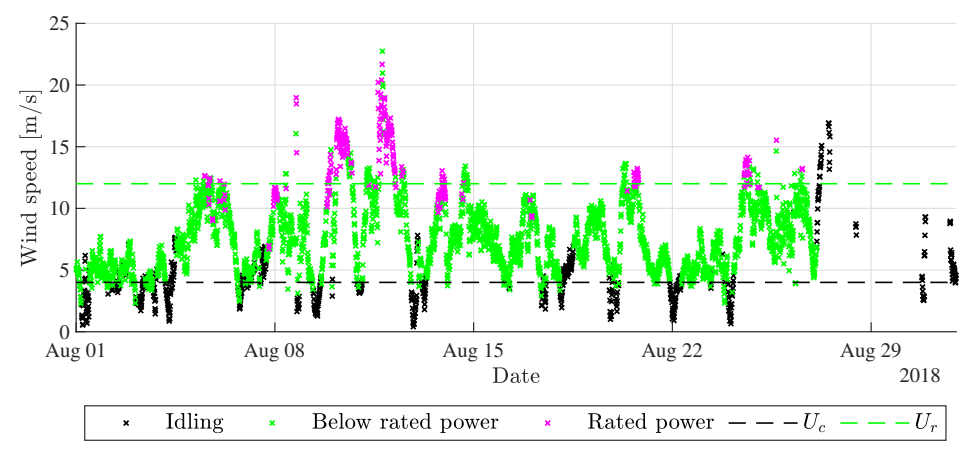

Figure 4: Wind speed and operational states of the turbine during the measurement campaign.

\section{System identification of the wind turbine substructure}

The input for the system identification analysis is the time series measured by the 7 accelerometers, resulting in $m=18$ channels $(4 \times 3$ directions in the TP and $3 \times 2$ directions in the tower). For each day, successive 30 min. data blocks are processed, resulting in maximum 48 time-frames per day. A separate system identification analysis is performed for each data block. The initial sampling frequency of $25 \mathrm{~Hz}$ is reduced to $5 \mathrm{~Hz}$ to focus on the global bending modes. A model order of 20 is used in the SSI-cov algorithm. This model order is found appropriate to capture the modes of interest.

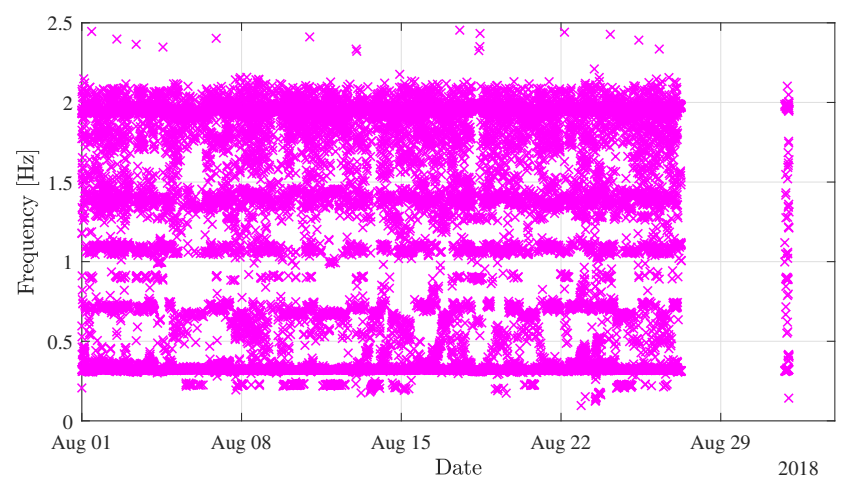

Figure 5: Identified frequencies (no post-processing).

Figure 5 presents frequencies estimated using the SSI-cov algorithm. One can observe that within the range of interest (up to approximately $2.5 \mathrm{~Hz}$ ) a large number of frequencies is identified. In order to identify poles representing the structural modes of interest, a classifier algorithm based on the modal assurance criterion (MAC) [24] and mode complexity estimate [25] has been applied. The algorithm calculates MAC values between the SSI-cov estimated modes and the numerical modes of interest. SSI-cov modes with MAC values above 0.80 are classified as structural, while the remaining are disregarded. Figure 6 presents a subset of frequencies classified as structural eigenfrequencies after applying the classifier algorithm with only the MAC criterion. Stable clusters of eigenfrequencies around 0.3 $\mathrm{HZ}$ and $2.0 \mathrm{~Hz}$ can be observed. They contain the 1 st and 2 nd global bending modes. The 1 st torsional mode is not identified, which presumably is due to the fact that the sensor setup was intended to capture global bending modes and not the torsional one (hence resulting in a high noise-to-signal ratio for the torsional mode). Consequently, only the global bending modes are considered in the remainder of the paper.

As the classifier does not distinguish between the fore-aft and the side-side modes, the clusters potentially contain both directions. Moreover, in Fig. 6, one can also observe a large number of additional frequencies in the range of 0.3-0.7 Hz. These modes are correlated with SCADA data and have been identified as P-frequencies originating from 


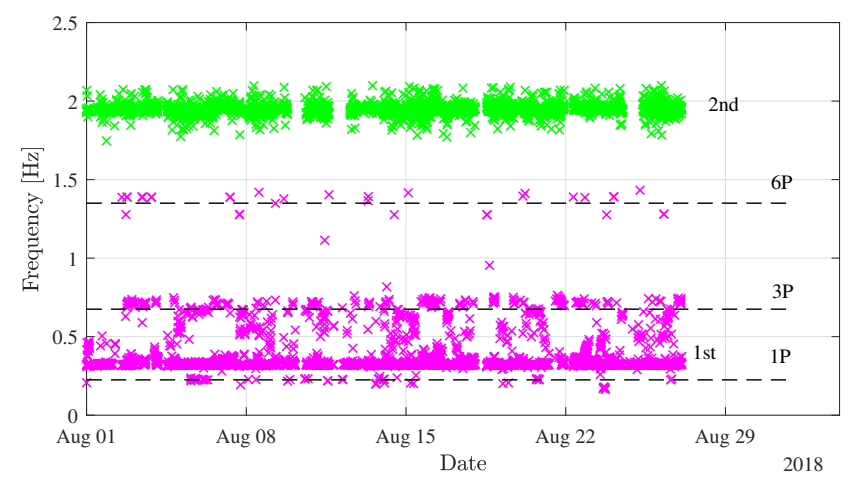

Figure 6: Identified frequencies processed with MAC classifier.

rotor revolution (1P), blades passing the tower (3P), and their harmonics (6P). The dashed lines in Fig. 6 represent the upper bound of the P-frequencies based on the rated rotor velocity. The P-frequencies are falsely classified as the 1st bending mode shape, as the mode shapes associated with the P-frequencies are geometrically indistinguishable (with the current sensor setup) from the 1st bending mode shape based on the MAC classifier only (variation in the wind pressure leads to varying wind force translated to the tower top).

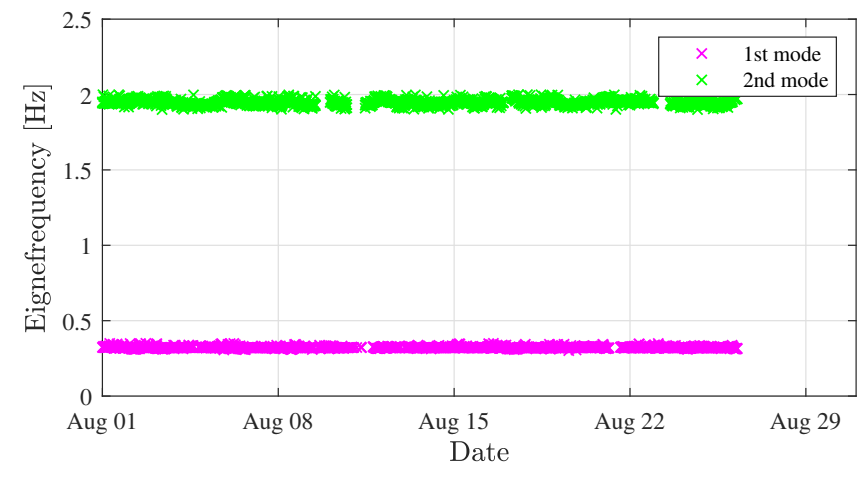

Figure 7: Identified eigenfrequencies after MAC classifier extended with complexity criterion.

To remove the P-frequencies from the results, the classifier algorithm is extended with the mode complexity criterion based on the Argand diagram [25]. The assumption is that the P-frequencies introduce non-classical damping, which results in complex modes. The complexity of the modes is estimated based on the imaginary part of the mode shapes, as proposed in [26]. Effectively, modes with high complexity around the expected P-frequency (estimated based on rotor revolution) are classified as P-frequencies and disregarded. Note that the P-frequencies are correlated with varying rotor revolution speed, hence the scatter between $1 \mathrm{P}$ and 3P. This scatter can be correlated with the rotor revolution from SCADA data and be used to further filter the P-frequencies. The complexity criterion can also be applied to further improve the classification of the remaining structural modes assuming that the mode complexity of these modes is low.

After applying the mode complexity criterion, a subset of identified eigenfrequencies including those of the 1st and 2nd global modes is obtained. Figure 7 depicts the subset, and one can observe that the P-frequencies are successfully filtered and the scatter in the 2nd eigenfrequency is reduced due to the removal of highly complex and hence potentially erroneous realizations. The mean values of the identified frequencies are $f_{1}=0.32 \mathrm{~Hz}$ and $f_{2}=1.95 \mathrm{~Hz}$, respectively. The results are derived based on both idling and operational states, covering all possible yaw directions and varying wind speeds. The coefficient of variation $(\mathrm{CoV})$ for the eigenfrequencies of the $1 \mathrm{st}$ and 2 nd global bending modes are $\mathrm{CoV} f_{1}=0.015$ and $\operatorname{CoV} f_{2}=0.017$, respectively. 
Table 1: Selection criteria for model updating input.

\begin{tabular}{cc}
\hline Assumptions & Criteria \\
\hline LTI system & no yawing, pitching \\
white-noise excitation & idling/parked condition \\
1st and 2nd modes & 4 eigenmodes identified \\
FA/SS symmetry planes & perpendicular modes \\
\hline
\end{tabular}

\section{Model updating of the wind turbine substructure}

\subsection{Numerical model}

The wind turbine structure and the jacket substructure are modelled using Ramboll's software ROSAP (Ramboll Offshore Structural Analysis Package), version 53 [27]. ROSAP is a general-purpose finite element package intended for both static and dynamic analysis of offshore structures. The jacket substructure is modelled with 3D Bernoulli-Euler beam elements [28], including the added mass of the surrounding water. The soil-structure interaction is modelled using a Winkler model [28] in which the steel pile elements are additionally supported by linearized springs representing the surrounding soil. The soil springs are linearized according to the API method [29]. The stiffness of the joints are modified to account for additional flexibility of the tubular joints according to Buitrago et al. [30]. The TP is modelled in a simplistic manner by a representative 3D Bernoulli-Euler beam system. The tower is modelled using 3D Bernoulli-Euler beam elements, including added masses representing internal secondary steel elements and flanges. The rotor-nacelle-assembly (RNA) comprising the nacelle, hub, and blades is modelled as a two-point mass system, including a mass moment of inertia tensor to reflect the difference in properties in the fore-aft and the side-side direction of the RNA. The parameters used in the initial numerical model are based on the information available in the design stage of the structure.

\subsection{Input for model updating}

The numerical model is updated in a setting where the eigenfrequencies and mode shapes of the first two bending modes are utilized as responses. The responses are estimated in the system identification Sec. 4. To discard realizations that severely violate the system identification assumptions, the selection criteria presented in Tab. 1 are employed. The first criterion excludes results with excessive yawing and pitching to comply with the LTI system assumption. The second criterion only includes results where the turbine is in idling or parked conditions to minimize the presence of non-white excitation stemming from wind and rotating machinery. Physical modes related to the foreaft and side-side directions are expected to be perpendicular due to a large stiffness disproportion in these directions. Therefore, similar properties are expected from the identified modes (criteria 3 and 4). After applying the 4 criteria, 9 sets containing modal parameters are selected as input for model updating.

The first criterion excludes results with excessive yawing and pitching to comply with the LTI system assumption. The second criterion only includes results where the turbine is in either the idling or the parked condition to minimize the presence of non-white excitation stemming from wind and rotating machinery. Due to a large stiffness disproportion in fore-aft and side-side directions, physical modes related to these directions are expected to be perpendicular. Therefore, similar properties are expected from the identified modes (criteria 3 and 4 ). After applying the above-mentioned criteria, 9 sets containing modal parameters are selected as input for model updating.

The uncertainties related to the selected modal parameter sets are quantified based on the eigenfrequencies' CoVs and are included in the weighting matrix, $\mathbf{W}_{\Lambda} . \operatorname{CoV} f_{1}=0.015$ and $\operatorname{CoV} f_{2}=0.017$ are assumed for the 1 st and 2nd bending eigenfrequencies, respectively. The uncertainties of the mode shapes are assumed to be tenfold of the respective eigenfrequencies CoVs, thus $\mathrm{CoV} m_{1}=0.15$ and $\mathrm{CoV} m_{2}=0.17$. The additional uncertainty is primarily attributed to the fact the uncertainties on both the state matrix and the output matrix are propagated to the mode shape estimates when using the SSI-cov method [23]. The uncertainty estimations applied here only account for a portion of the measurement and statistical uncertainties related to these quantities. However, as mentioned in Subsec. 2.3, the scope of the paper is to update the mean values of the parameters, thus such level of details is deemed sufficient. 
Table 2: Sensitivity and uncertainty matrix for model parameters.

\begin{tabular}{cccccc}
\hline Parameter & \multicolumn{5}{c}{ Uncertainty } \\
Type & Symbol & Metric & CoV & Reference & \multirow{2}{*}{ Sensitivity } \\
\hline Jacket stiffness* & $\mathrm{k}_{j}$ & 1 & 0.05 & {$[31]$} & 1 \\
Turbine stiffness* & $\mathrm{k}_{t}$ & 2 & 0.25 & $* *$ & 2 \\
TP stiffness* & $\mathrm{k}_{T P}$ & 2 & 0.25 & $* *$ & 2 \\
Piles stiffness* & $\mathrm{k}_{p}$ & 1 & 0.10 & $* *$ & 2 \\
Joint stiffness* & $\mathrm{LJF}$ & 2 & 0.50 & {$[32]$} & 1 \\
Leg diameter & $\mathrm{D}_{l}$ & 0 & - & - & 2 \\
Leg thickness & $\mathrm{t}_{l}$ & 1 & - & - & 1 \\
Brace diameter & $\mathrm{D}_{b}$ & 0 & - & - & 1 \\
Brace thickness & $\mathrm{t}_{b}$ & 1 & - & - & 0 \\
Scour* & $\mathrm{sc}$ & 2 & - & - & 1 \\
Corrosion* & $\mathrm{corr}$ & 2 & - & - & 2 \\
Marine growth* & $\mathrm{mg}$ & 2 & - & - & 2 \\
added mass* & $\mathrm{a}_{m}$ & 2 & 0.50 & $* *$ & 1 \\
hub mass* & $\mathrm{m}_{h}$ & 1 & 0.02 & {$[33]$} & 3 \\
lateral soil stiffness* & $\mathrm{py}$ & 3 & 1.00 & {$[34,35]$} & 1 \\
axial soil stiffness* & $\mathrm{tz}$ & 3 & 1.00 & {$[35,35]$} & 2 \\
pile tip stiffness* & $\mathrm{qw}$ & 3 & 1.00 & {$[36]$} & 0 \\
\hline
\end{tabular}

Metric (uncert./sensit.): 0- negligible, 1- low, 2- medium, 3- high

* Parameters to be updated; Uncertainty estimation: ** Engineering judgement

\subsection{Physical parameters to be updated}

The number of physical parameters included in a numerical model can become large and updating all of them can, for many practical applications, result in an ill-posed optimization problem. Thus, some criteria for selecting a subset of parameters to be updated must be implemented. Below, such an attempt is presented based on the principle of Fisher information (sensitivity vs. uncertainty) supplemented with engineering judgment. The assumption is to update parameters that are highly uncertain and, in the same time, affect the chosen modal parameters (frequencies and modes).

To select which model parameters should be updated, their sensitivities to modal parameters are quantified. This study involves modifying each model parameter in the numerical model independently and extracting the shifted modal parameters. Model parameters whose alterations result in high shifts in the modal parameters are assigned a high sensitivity metric, while model parameters whose alterations do not yield a substantial modal parameter shift are assigned a low sensitivity metric. Subsequently, the uncertainties of the parameters are determined in a separate study. Highly uncertain parameters are assigned a high uncertainty metric (e.g., CoV, assuming no bias), while parameters believed to be estimated with high precision are assigned a low uncertainty metric. Such analysis has been performed and the results are presented in Tab. 2. A large set of parameters is initially screened to select potential candidates for model updating. The parameters with medium or high sensitivity towards global modes are soil stiffness, corrosion, marine growth, hub mass, turbine stiffness, and TP stiffness. The most uncertain parameters (metric 2 or 3) are expected to be turbine stiffness, TP stiffness, joint stiffness, scour, corrosion, marine growth, added mass, and, especially, soil stiffness. By selecting highly uncertain and/or highly sensitive parameters, 10 are considered for updating. These are indicated by the asterisk in Tab. 2 .

The generic uncertainty metric from Tab. 2 is now supplemented with CoV of the selected parameters. The uncertainties are estimated based on a literature review, supplemented with engineering judgement where no reference could be found. The jacket stiffness is assumed to be varied via Young's modulus of the material used. The CoV is well-described and found to be 0.05 [31]. Therefore, this value is used. The turbine stiffness represents the stiffness of the structure above the interface including tower, RNA, and blades. As a simplified model is used for this superstructure large uncertainty of 0.25 is assumed based on engineering judgement. The uncertainty associated with the pile and TP stiffness is a combination of a Young's modulus uncertainty and any modelling uncertainties due to 
Table 3: Estimated and model-predicted eigenfrequencies.

\begin{tabular}{lcccc}
\hline \multirow{2}{*}{ Configuration } & \multicolumn{2}{c}{$f_{1}[\mathrm{~Hz}]$} & \multicolumn{2}{c}{$f_{2}[\mathrm{~Hz}]$} \\
\cline { 2 - 5 } & FA & SS & FA & SS \\
\hline Estimated (measurements) & 0.32 & 0.32 & 1.96 & 1.99 \\
Design (model) & 0.30 & 0.30 & 1.37 & 1.40 \\
As-built (model) & 0.31 & 0.31 & 1.57 & 1.63 \\
Updated (model) & 0.32 & 0.32 & 1.94 & 2.00 \\
\hline
\end{tabular}

simplifications in the modelling approach, e.g., beam vs. shell elements and PY soil curves vs. 3D soil model. Thus, the initial 0.05 value is increased to 0.10 and 0.25 for the pile and TP elements, respectively. The increase accounts for uncertainty in modelling of a pile element with contribution from the structural component (steel) and surrounding soil. The uncertainty for the TP element is increased to account for the simplified beam representation used.

The uncertainty on the hub mass is very low as the element is weighed prior to installation, thus a value of 0.02 is assumed based on the available as-built weight reports [33]. The added mass parameter represents the added mass contribution on the submerged part of the jacket substructure due to the surrounding water. It is modelled as a multiplication factor of the initially estimated added mass. The added mass uncertainty depends on the shape of the structural element and its orientation, which changes as waves pass. To reflect these uncertainties, a value of 0.50 is chosen. The local joint flexibility representing the effective stiffness of a tubular joint is modelled via the simplified Buitrago equations [30]. This approach is known to be imprecise [32], so to account for this uncertainty, a value of 0.50 is chosen. The soil stiffness is modified via correction parameters, py, tz, qw, which update the initial stiffness of the linearized API curves [29]. The soil stiffnesses are considered to be the most uncertain parameters, thus a CoV of 1.00 is assumed for these. The value is chosen based on literature studies regarding soil uncertainty estimation $[34,36,35]$.

In this study, boundaries are imposed on the model parameters to be updated to reflect physical limitations of the mass and stiffness, i.e., only positive values are allowed. Other boundaries can also be adopted, e.g., the validity range of the parameters provided in guidelines/standards.

\subsection{Results}

In this subsection, model updating results are presented. The update is performed using the one modal parameter set which is the most consistent with the selection criteria defined in Subsec. 5.2. The frequencies and MAC results for different stages of the update are presented in Tab. 3 and Tab. 4. The discrepancies between the initial (design) model and the estimated eigenfrequencies are $6 \%$ and $30 \%$ for the 1st and 2nd global bending mode, respectively. The corresponding MAC values are 0.96 and 0.85 .

The model updating is performed in two steps. The first step involves updating the parameters based on the as-built reports. These reports are normally available after the installation is completed. They include some information like the as-built weight of the structure and information from pile driving (e.g., pile stick-up). In this stage of the update, it is also assumed that no scour, corrosion, or marine growth have developed. This assumption is based on the fact that the measurement campaign was performed 1 year after the installation, thus it is assumed that these processes did not have enough time to initiate in the specific environment. After including this information, the frequency discrepancies are reduced to 3\% and 20\% for the 1 st and 2nd global modes, with MAC values of 0.97 and 0.98 . Finally, the model updating procedure based on measurement data is performed as described in Subsec. 2.2. After the update, the discrepancy between the measured and modelled 1 st global frequency is reduced from the initial $6 \%$ to $0.3 \%$. The discrepancy in the 2 nd global frequency is reduced from the initial $30 \%$ to $1.0 \%$. The MAC value after the update is 0.96 and 0.99 for the 1 st and 2nd global mode, respectively.

The parameter set obtained after the model updating is presented in Tab. 5 . The jacket stiffness, $k_{j}$, the structural pile stiffness, $k_{p}$, the hub mass, $m_{h}$, and the local joint flexibility, LJF, remain almost unchanged after the update. Substantial updating is observed in the TP stiffness, $k_{T P}$, with a reduction of $64 \%$. The TP stiffness change is not surprising, as the simplified beam modelling strongly depends on the estimation of the representative stiffness, which can be difficult to assess without a detailed 3D model. A substantial update is also observed in the turbine stiffness, 
Table 4: Estimated and model-predicted MAC.

\begin{tabular}{lcccc}
\hline \multirow{2}{*}{ Configuration } & \multicolumn{2}{c}{ Mode $1[-]$} & \multicolumn{2}{c}{ Mode 2 [-] } \\
\cline { 2 - 5 } & FA & SS & FA & SS \\
\hline Estimated (measurements) & 1.00 & 1.00 & 1.00 & 1.00 \\
Design (model) & 0.96 & 0.98 & 0.85 & 0.93 \\
As-build (model) & 0.97 & 0.99 & 0.98 & 0.99 \\
Updated (model) & 0.96 & 0.98 & 0.99 & 0.99 \\
\hline
\end{tabular}

Table 5: Updated mean values of the selected model parameters.

\begin{tabular}{cccccc}
\hline \multicolumn{2}{c}{ Parameter } & & \multicolumn{3}{c}{ Value } \\
Type & Symbol & Unit & Initial & Updated & Normalized \\
\hline Jacket stiffness & $k_{j}$ & - & $1.00 \mathrm{e}+0$ & $9.95 \mathrm{e}-1$ & 0.99 \\
Turbine stiffness & $k_{t}$ & - & $1.00 \mathrm{e}+0$ & $1.83 \mathrm{e}+0$ & 1.83 \\
TP stiffness & $k_{T P}$ & - & $1.00 \mathrm{e}+0$ & $3.41 \mathrm{e}-1$ & 0.36 \\
Pile stiffness & $k_{p}$ & - & $1.00 \mathrm{e}+0$ & $1.02 \mathrm{e}+0$ & 1.02 \\
Added mass & $a_{m}$ & - & $1.00 \mathrm{e}+0$ & $0.84 \mathrm{e}+0$ & 0.84 \\
Hub mass & $m_{h}$ & $\mathrm{~kg}$ & $1.44 \mathrm{e}+5$ & $1.47 \mathrm{e}+5$ & 1.01 \\
lateral soil stiffness & py & - & $1.00 \mathrm{e}+0$ & $1.33 \mathrm{e}-1$ & 0.13 \\
axial soil stiffness & $\mathrm{tz}$ & - & $1.00 \mathrm{e}+0$ & $6.96 \mathrm{e}-2$ & 0.07 \\
pile tip stiffness & $\mathrm{qw}$ & - & $1.00 \mathrm{e}+0$ & $6.62 \mathrm{e}-1$ & 0.66 \\
Joint stiffness & $\mathrm{LJF}$ & - & $1.00 \mathrm{e}+0$ & $1.02 \mathrm{e}+0$ & 1.02 \\
\hline
\end{tabular}

Table 6: Pile head stiffness update.

\begin{tabular}{ccccc}
\hline & \multicolumn{2}{c}{$k_{x y}[\mathrm{kN} / \mathrm{m}]$} & \multicolumn{2}{c}{$k_{z}[\mathrm{kN} / \mathrm{m}]$} \\
Configuration & Value & Ratio & Value & Ratio \\
\hline Design & $2.8 \mathrm{e}+5$ & 1.0 & $1.0 \mathrm{e}+6$ & 1.0 \\
As-built & $4.4 \mathrm{e}+5$ & 1.6 & $1.3 \mathrm{e}+6$ & 1.3 \\
Updated & $1.3 \mathrm{e}+6$ & 4.7 & $6.0 \mathrm{e}+6$ & 6.0 \\
\hline
\end{tabular}


Table 7: Scatter in the updated mean values of the model parameters for varying sets of modal parameters.

\begin{tabular}{cccc}
\hline Parameter & Unit & Mean & CoV of mean value \\
\hline$k_{j}$ & - & $9.95 \mathrm{e}-1$ & $<0.01$ \\
$k_{t}$ & - & $1.92 \mathrm{e}+0$ & 0.04 \\
$k_{T P}$ & - & $3.30 \mathrm{e}-1$ & 0.06 \\
$a_{m}$ & - & $8.59 \mathrm{e}+0$ & 0.05 \\
$m_{h}$ & $\mathrm{~kg}$ & $1.47 \mathrm{e}+5$ & $<0.01$ \\
$k_{x y}$ & $\mathrm{kN} / \mathrm{m}$ & $1.18 \mathrm{e}+6$ & 0.12 \\
$k_{z}$ & $\mathrm{kN} / \mathrm{m}$ & $5.50 \mathrm{e}+6$ & 0.11 \\
$\mathrm{LJF}$ & - & $1.02 \mathrm{e}+0$ & 0.02 \\
\hline
\end{tabular}

$k_{t}$. The stiffness is increased by $83 \%$. Large update of this parameter can be explained by the fact that a low-fidelity turbine model (RNA and blades) is used. Its representative parameters are inferred from implicit, confidential data (blade moments of inertia) and thus prone to imprecise estimation. Worth noting is the significant update of the soil parameters. While the py, tz, qw parameters are convenient from a modelling perspective, they are difficult to interpret on a physical level. Thus, additional results have been extracted, namely, the pile head stiffness in the lateral and the axial direction, $k_{x y}$ and $k_{z}$, respectively. The results are presented in Tab. 6 . The soil stiffness is increased by a factor of 4.7 and 6.0 for the lateral and axial directions, respectively. An elaborate discussion on the profound update of the TP, turbine, and soil stiffnesses is provided in Sec. 6.

\subsection{Sensitivity of the updating procedure}

As the estimated modal parameters exhibit some scatter $\left(\mathrm{CoV}_{f 1}=0.015\right.$ and $\left.\mathrm{CoV}_{f 2}=0.017\right)$, it is investigated how sensitive the updated parameters are towards variation in the modal parameters. To investigate this effect, the estimated modal parameters from 9 different idling time-frames, see Sec. 5.2, are used to perform model updating. The model updating setup uses the same starting values for the parameters and the same weighting matrices, $\mathbf{W}_{\Lambda}$ and $\mathbf{W}_{\Theta}$; effectively altering only the input modal parameters. The scatter in the updated mean values of the model parameters is presented in Tab. 7. The scatter is reported as $\mathrm{CoV}$ on the updated mean values of the parameters. The updated values for jacket stiffness and hub mass present negligible scatter $(\mathrm{CoV}<0.01)$. The turbine stiffness, $k_{t}$, TP stiffness, $k_{T P}$, and added mass, $a_{m}$, exhibit low scatter, namely, CoV of $0.04,0.06$, and 0.05 . Finally, the soil stiffness $\mathrm{CoV}$ are 0.12 and 0.11 for $k_{x y}$ and $k_{z}$, respectively. The mean value of the updated parameters based on 9 modal parameter sets, see Tab. 7, are close to, yet not identical with, the updated parameters provided in Tab. 5.

\section{Discussion}

This section offers a discussion of the model updating procedure and appertaining results presented in Sec. 4 and Subsec. 5.4. Particular focus is assigned to the initial deviation in the 2 nd bending eigenfrequency and the profound update of selected model parameters.

\subsection{On the initial eigenfrequency discrepancy}

Based on the system identification results provided in Sec. 4 and the corresponding model predictions presented in Subsec. 5.1, a discrepancy of 30\% was reported for the 2nd bending eigenfrequency. Part of that discrepancy can be explained by merely stating that the two estimations aim to describe different stages of the same ageing structure. The numerical model is based on the design parameters, which, according to the design recommendations [37], are representative for the averaged conditions during the lifetime of the structure, typically 25 years. However, the in-situ estimation is derived based on the structural condition 1 year after installation. This is especially relevant in the context of phenomena accumulating over time, e.g., corrosion and marine growth, as these affect the discussed eigenfrequency. After adjusting the numerical model to a representative 1 year condition, the eigenfrequency discrepancy is reduced to $20 \%$. The bulk of the remaining discrepancy can be attributed to the misprediction of the soil stiffness. It is commonly accepted in the industry that the soil stiffness estimation is highly uncertain [34, 36, 35] and different measures are currently being explored to improve the accuracy of soil stiffness models [38]. As an example, a recent publication 
[39] investigated the effect of soil stiffness modelling uncertainty on the eigenfrequencies of an offshore wind jacket substructure. The results estimated based on a number of state-of-the-art procedures were subsequently compared to the in-situ estimations, and discrepancies of up to $40 \%$ were reported. In order to reduce the discrepancies, a substantial soil stiffness update was proposed, which is in line with the updating results documented in the present study.

\subsection{On the update of the turbine, TP, and soil stiffnesses}

In Subsec. 5.4, the updated model parameters are stated, and it is found that substantial updating of the turbine stiffness, TP stiffness, and soil stiffness is required. Since the soil stiffness has been addressed in Subsec. 6.1, the present subsection discusses the updated turbine and TP stiffnesses. As elaborated in Subsec. 5.1, the numerical models of the turbine and the TP are simplified. The turbine's two-point mass system neglects the stiffness of the blades and their potential coupling to the substructure, especially to the brace elements. The simplified beam model of the TP is calibrated to represent global bending stiffness properties of the structure, while neglecting local effects. Despite the mentioned shortcomings, the simplified modelling approaches have been taken in this paper due to the following reasons; 1) state-of-the-art modelling approaches are used and 2) high-fidelity models are often not available due to practical and confidentiality reasons. Below, a few remarks on the high-fidelity models and the updated parameters are provided.

Worth noting is the fact that the turbine stiffness and the TP stiffness are updated in opposite directions, i.e., a reduction of the TP stiffness and an increase of the turbine stiffness. Since both of these parameters affect the bending stiffness in the same direction, a positive correlation of the updated parameters could be expected. However, the initial value of the parameters is derived independently. Consequently, depending on the precision of the initial estimates, one can imagine a situation where the two parameters after the update can be positively correlated (given both initial stiffnesses are simultaneously either under- or over-estimated) or, as presented in this paper, where the parameters are updated in the opposite directions (given the initial stiffnesses are over- and under-estimated).

High-fidelity modelling of the TP using shell and/or solid finite elements would result in a TP model of a size of at least an order of magnitude larger than the entire remaining model. Assuming each element would introduce two parameters to be updated (mass and stiffness), such a TP model would result in an infeasible number of parameters to be updated. Modelling of the turbine, including an explicit beam formulation of the blades, is possible in a number of commercial aeroelastic software, e.g., FAST [40], Bladed [41], and FLEX [42]. By using such software (in combination with access to turbine data) a high-fidelity turbine model can be obtained, where the coupling between the braces and the blades is included. However, access to such data is typically restricted to wind turbine vendors. As a result, these models are not widely available. Moreover, the brace-blade coupling is not expected to be of crucial importance for model updating when low-order, global bending modes are used as responses (note that the 1st bending mode is dominated by the tower top displacement, while the 2nd bending mode is dominated by lateral TP displacement). On the contrary, brace-blade coupling can become critical if higher-order mode shapes dominated by the local brace displacements are included. Consequently, it is deemed impractical, at least in the presented framework and design procedures, to include high-fidelity TP and turbine models. Nevertheless, a resulting substantial update of the TP and turbine parameters might indicate insufficient precision of the modelling approach implemented in this study or imprecise initial estimation of the parameters related to the simplified models.

Alternatively, one can perform model updating of the substructure alone. The merit of this approach lays in removing the uncertainties related to the turbine modelling, consequently increasing sensitivity to the remaining highly uncertain parameters, namely, those associated with the soil stiffness. However, this approach has two main limitations; 1 ) it is only applicable to new structures instrumented prior to commissioning and 2) it introduces an additional complication in the installation schedule to obtain comprehensive measurement data.

\section{Conclusions}

This paper demonstrates the application of model updating in the context of an offshore wind jacket substructure exposed to both environmental and operational variability. It is shown how the sensitivity-based model updating, employing a subset of experimentally estimated modal parameters as responses, enables updating of physical parameters within the model to more accurately reflect in-situ conditions. For the considered example, the initial maximum 
eigenfrequency discrepancy of $30 \%$, between the experimental findings and the model-predicted ones, is reduced to $1 \%$ after the update. The bulk of the initial discrepancy is attributed to a highly uncertain soil stiffness estimation.

The present study is limited to 1) estimating responses from idling states of the turbine, 2) using global modes of the structure as responses, 3) updating mean values of the physical parameters, and 4) using simplified models of the TP and the turbine. The first limitation is related to the chosen system identification method, the second is the consequence of the given sensor setup, the third one is characteristic for the sensitivity-based updating scheme, while the last one is due to practical/confidential reasons. Regardless of the mentioned limitations, the presented framework is general and its applicability for in-situ data is demonstrated in the paper.

\section{Acknowledgements}

The work presented herein is financially supported by Ramboll Foundation, Ramboll Energy, and Innovation Fund Denmark. This project has received funding from the European Union's Horizon 2020 research and innovation program under grant agreement No. 745625 (ROMEO) ("Romeo Project" 2018). The financial support is highly appreciated. The first author would like to thank colleagues from Ramboll for their continuous support.

\section{References}

[1] U.S. Department of Energy, 2018 Offshore Wind Technologies Market Report, Tech. rep. (2018).

[2] Global Wind Energy Council, Global Wind Report 2018, Tech. rep. (2018).

[3] BSH-7005, Standard Design. Minimum requirements concerning the constructive design of offshore structures within the Exclusive Economic Zone (EEZ), Tech. rep. (2015).

[4] M. V. Grieves, Virtually Intelligent Product Systems: Digital and Physical Twins, 2019. doi : 10.2514/5.9781624105654.0175.0200. URL https://arc.aiaa.org/doi/abs/10.2514/5.9781624105654.0175.0200

[5] W. Kritzinger, M. Karner, G. Traar, J. Henjes, W. Sihn, Digital twin in manufacturing: A categorical literature review and classification, in: Proceedings of the 16th Symposium on Information Control Problems in Manufacturing, INCOM 2018, 2018, pp. 1016 - 1022. doi : https: $/ /$ doi.org/10.1016/j.ifacol.2018.08.474

[6] U. T. Tygesen, M. Jepsen, J. Vestermark, N. Dollerup, A. Pedersen, The true digital twin concept for fatigue re-assessment of marine structures, in: Proceedings of the 37th International Conference on Ocean, Offshore and Arctic Engineering, OMAE2018, 2018. doi: 10.1115/OMAE2018-77915.

[7] D. Augustyn, U. T. Tygesen, M. D. Ulriksen, J. D. Sørensen, Data-driven design and operation of offshore wind structures, in: Proceedings of the 29th International Offshore and Polar Engineering Conference, ISOPE2019, 2019, pp. 491-498. URL https://onepetro.org/conference-paper/ISOPE-I-19-618

[8] D. Tcherniak, S. Chauhan, M. Hansen, Applicability Limits of Operational Modal Analysis to Operational Wind Turbines, Springer New York, 2011. doi:https://doi.org/10.1007/978-1-4419-9716-6_29.

[9] U. T. Tygesen, K. Worden, T. Rogers, G. Manson, E. Cross, State-of-the-Art and Future Directions for Predictive Modelling of Offshore Structure Dynamics Using Machine Learning, Springer International Publishing, 2019. URL https://link.springer.com/chapter/10.1007/978-3-319-74421-6_30

[10] M. W. Häckell, R. Rolfes, Monitoring a $5 \mathrm{mw}$ offshore wind energy converter-condition parameters and triangulation based extraction of modal parameters, Mechanical Systems and Signal Processing 40 (2013) 322 - 343. doi : https://doi.org/10.1016/j.ymssp. 2013. 04.004 .

[11] C. Devriendt, F. Magalhães, W. Weijtjens, G. Sitter, Cunha, P. Guillaume, Structural health monitoring of offshore wind turbines using automated operational modal analysis, Structural Health Monitoring 13 (6) (2014) 644-659. doi:10.1177/1475921714556568.

[12] W. Popko, F. Vorpahl, P. Antonakas, Investigation of local vibration phenomena of a jacket sub-structure caused by coupling with other components of an offshore wind turbine, in: Proceedings of the 23rd International Offshore and Polar Engineering Conference, ISOPE2013, 2013, pp. 491-498.

URL https://www.onepetro.org/conference-paper/ISOPE-I-13-012

[13] M. I. Friswell, J. E. Mottershead, Finite element model updating in structural dynamics, Kluwer Academic Publishers, Dordrecht, 1995.

[14] D. Bernal, M. D. Ulriksen, Output feedback in the design of eigenstructures for enhanced sensitivity, Mechanical Systems and Signal Processing 112 (2018) 22 -30. doi:https://doi.org/10.1016/j.ymssp. 2018.04.032.

[15] E. Patelli, Y. Govers, M. Broggi, H. Gomes, M. Link, J. E. Mottershead, Sensitivity or bayesian model updating: a comparison of techniques using the dlr airmod test data, Archive of Applied Mechanics 87 (5) (2017) 905-925. doi:10.1007/s00419-017-1233-1.

[16] C. Schedlinski, F. Wagner, K. Bohnert, J. Frappier, A. Irrgang, R. Lehmann, A. Muller, Experimental modal analysis and computational model updating for a car body in white, in: Proceedings of the 1st International Conference on Noise and Vibration Engineering, ISMA2004, 2004.

[17] M. Luczak, S. Manzato, B. Peeters, K. Branner, P. Berring, M. Kahsin, Updating finite element model of a wind turbine blade section using experimental modal analysis results, Shock and Vibration 2014 (2014). doi:10.1155/2014/684786.

[18] P. van Overschee, L. de Moor, Subspace identification for linear systems: theory, implementation, applications, Kluwer Academic Publishers, 1996. doi : 10.1007/978-1-4613-0465-4. 
[19] C. Devriendt, F. Magalhaes, W. Weijtjens, G. De Sitter, A. Cunha, P. Guillaume, Automatic identification of the modal parameters of an offshore wind turbine using state-of-the-art operational modal analysis techniques, in: Proceedings of the 5th International Operational Modal Analysis Conference, IOMAC 2013, 2013.

[20] J. E. Mottershead, M. Link, M. I. Friswell, The sensitivity method in finite element model updating: A tutorial, Mechanical Systems and Signal Processing 25 (7) (2011) 2275 - 2296. doi : https : //doi.org/10.1016/j.ymssp.2010.10.012.

[21] R. Willoughby, Solutions of ill-posed problems (a. n. tikhonov and v. y. arsenin), SIAM Review 21 (1979) 266-267. doi : 10.1137/1021044.

[22] J. D. Sørensen, H. S. Toft, Probabilistic design of wind turbines, Energies 3 (2010) 241-257. doi:10.3390/en3020241.

[23] E. Reynders, R. Pintelon, G. Roeck, Uncertainty bounds on modal parameters obtained from stochastic subspace identification, Mechanical Systems and Signal Processing 22 (4) (2008) 948 - 969, special Issue: Crack Effects in Rotordynamics. doi : https ://doi .org/10.1016/ j.ymssp. 2007.10.009.

[24] A. J. Allemang, The modal assurance criterion (mac): Twenty years of use and abuse, Journal of Sound and Vibration 37 (2003) 14-21.

[25] M. Imregun, D. Ewins, Complex modes - origins and limits, in: Proceedings of the 13th International Modal Analysis Conference, IMAC $1995,1995$.

[26] P. Olsen, A. Skafte, J. Hansen, M. Hovgaard, R. Brincker, C. Ventura, Identification of systems with complex modes using oma, in: Proceedings of the 5th IOMAC International Operational Modal Analysis Conference, 2013.

[27] Ramboll, ROSAP - Ramboll Offshore Structural Analysis Package, version 53, Tech. rep. (2018).

[28] R. D. Cook, D. S. Malkus, M. E. Plesha, R. J. Witt, Concepts and Applications of Finite Element Analysis, 4th Edition, John Wiley \& Sons, 2001.

[29] API-RP-2A, Recommended Practice for Planning, Designing and Constructing Fixed Offshore Platforms, Tech. rep. (2014).

[30] J. Buitrago, B. Healy, T. Chang, Local joint flexibility of tubular joints, in: Proceedings of the 12th International Conference on Offshore Mechanics and Arctic Engineering, OMAE 1993, 1993.

[31] A. Sadowski, J. Rotter, T. Reinke, T. Ummenhofer, Statistical analysis of the material properties of selected structural carbon steels, Structural Safety 53 (2015) 26 - 35. doi : https ://doi.org/10.1016/j . strusafe.2014.12.002.

[32] M. B. Nielsen, J. F. Jensen, C. Harper, L. Knudsen, R. R. Pedersen, State-of-the-art framework for structural design of offshore wind jacket foundations, in: Proceedings of the 1st Nordic Steel Construction Conference, Nordic Steel 2018, 2019, pp. 817-822. doi:10.1002/cepa. 1139.

[33] Confidential author, As-Built Report, Tech. rep. (2016).

[34] V. Negro, J. López-Gutiérrez, M. Esteban, C. Matutano, Uncertainties in the design of support structures and foundations for offshore wind turbines, Renewable Energy 63 (2014) 125 - 132. doi : https://doi.org/10.1016/j.renene.2013.08.041.

[35] B. Yeter, Y. Garbatov, C. Soares, Uncertainty analysis of soil-pile interactions of monopile offshore wind turbine support structures, Applied Ocean Research 82 (2019) 74 - 88. doi : https : //doi.org/10.1016/j . apor.2018.10.014.

[36] A. Bertossa, Evaluating geotechnical uncertainty for Offshore Wind Turbine foundation design at St. Brieuc Wind Farm. doi : 10.1680/ ecsmge.60678.vol3.178.

[37] DNVGL-ST-0126, Support structures for wind turbines, Tech. rep. (2016).

[38] Byrne, B. W., Burd, H. J., Zdravkovi'c, L., McAdam, R. A., Taborda, D. M. G, Houlsby, G. T., Jardine, R. J., Martin, C. M., Potts, D. M., Gavin, K. G., Pisa: new design methods for offshore wind turbine monopiles, Revue Francaise de Geotechnique 158 (2019). doi: 10.1051/geotech/2019009.

[39] L. Bom, S. Siedler, J. Tautz-Weinert, Validation of cpt-based initial soil stiffness in sand for offshore wind jacket piles, in: Proceedings of the Messen in der Geotechnik, 2020.

[40] Jonkman J. M., Buhl M. L., FAST User's Guide-Updated August 2005, Tech. rep. (2005).

[41] DNVGL, Wind turbine design software - bladed (2020).

URL https://www.dnvgl.com/services/wind-turbine-design-software-bladed-3775

[42] Øye S., Various FLEX 5 documentation, Tech. rep. (2001) 